\title{
Comparative Analysis of Global Feature Extraction Methods for Off-line Signature Recognition
}

\author{
Hifzan Ahmed \\ Student, Department of Electrical Engineering \\ Jabalpur Engineering College \\ Jabalpur, India
}

\author{
Shailja Shukla \\ Phd, Professor, Department of Electrical \\ Engineering \\ Jabalpur Engineering College \\ Jabalpur, India
}

\begin{abstract}
In this paper, a method is proposed for feature extraction of offline signature recognition system. The proposed method is based on global features to identify forgeries and also median filter is introduces for noise reduction. The Proposed feature extraction method is compared with Discrete Radon Transform (DRT). Both the feature extraction method extracts one dimensional global features and the alignment between features is performed by Dynamic Time Warping (DTW). When being trained using 6 genuine signatures of each person and 250 forgeries taken from our database, the proposed method obtained an equal error rate (EER) of $8.40 \%$. The false acceptance rate (FAR) for proposed method was also kept as low as $8.80 \%$.
\end{abstract}

\section{Keywords}

Median filter, Global features, Discrete Radon transform, Dynamic time warping.

\section{INTRODUCTION}

Signatures are used as a source to authenticate and also as a authorized identity of the writer. By the Fraud and forgery of person's signature any unwanted person can get access of that particular person's valuable assets. To prevent this type of fraud or forgery a recognition or verification system is needed that can analyze, verify and identify signature's patterns. We use signatures every day to legalized or authorize bank transactions, credit card transactions, driving license, property documents and contracts. The Transaction of billions or trillions of rupees and dollars can be done easily only by making ours signature or any banks bill or cheque. Bank and financial organizations primarily focus on the pattern of our signature for verification purposes, when we sign any document.

Signature verification or recognition is the process of verifying one input of a person against one input in the database. The Genuine signature from the data base is shown in Figure 1(a) and forgery for the same signature is shown in Figure 1(b). We can classify Signature recognition system by two ways i.e off-line (static) and on-line (dynamic). In these two methods on-line has disadvantage because it requires the person whose signature is verified. This cannot be applied in many cases where the person whose signature is to be verified is absent. Thus off-line method of signature verification is accepted a choice for universal application. Off-line techniques use only the image of the information for signature recognition. On-line techniques use both the shape of the signature as well as dynamic information such as speed, time taken etc. Offline systems are more applicable and easy to use in comparison with on-line systems. But in many parts of the world however it is considered more difficult than on-line verification due to the lack of dynamic information [1].

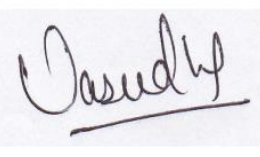

(a)

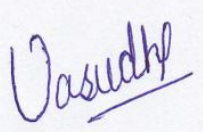

(b)
Fig 1: (a) Genuine Signature, (b) Forged signature

A signature by any person or authority is considered to be the seal of approval and remains the most preferred means of authentication and validation. Now days, check fraud has increased gradually. While off-line (static) signature verification is the most suited technique for reducing fraud through payment from checks, fax money transactions, payment orders etc. For signature verification and recognition system, a signature cannot be too long or too short. If a signature is too long, too much behavioral data will be presented. As a result, it will be difficult for the Signature verification and recognition system to identify consistent and unique data points. If a signature is too short, insufficient data will be captured, giving rise to a higher False Accept Rate and False Rejection Rate.

The verification and recognition system can be divided mainly in four steps i.e. pre-processing, feature extraction, data training and verification. The pre-processing is done to simplify subsequent operations without losing relevant information and the purpose of feature extraction is to reduce the data by measuring certain feature or properties. The data of feature extraction is used to train the system and for verification [2]. The data obtain from the signature extraction image by feature depends on feature types i.e Global and Local features. The problems of recognition process are reduced by extracting important features of the signature [3]. Global features which are extracted from the whole signature image and local features are extracted from the small portion of signature image. The global features can be extracted easily and are robust to noise. On the other hand, local features provide rich descriptions of writing shapes but for the reliable local features are still difficult to extract. The global features based approaches are more popular in off-line verification and recognition system.

The rest of the paper is structured as follows. In sections II, Pre-processing of signature verification systems are presented. In section III, two different features extraction methodological details are summarized. Section IV, discusses system training implementation details. Section V, verification part of the system is described. Finally, the Experimental results and conclusion are drawn in sections VI and VII respectively. 


\section{PREPROCESSING}

In image representation one is concerned with characterization of the quantity that each picture-element (also called pixel) represent [4]. The input signature image as shown in Figure 2(a) is obtained by scanning signature of the person. The image obtained is then converted into gray-scale image, and then the image is further pre-processed i.e. it is binarized. The binary image of the signature contains only 0 's and 1's. Where 0's represents the signature boundary and 1's represents the blank white area or the background region as shown in Figure 2(b). This is done by specifying a specific threshold, above which every gray value is 1 and below which every gray value is 0 . Then we take inverse of the binary image, where 0's represents black area or background and 1's represents signature boundary as shown in Figure 2(c).

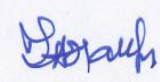

(a)

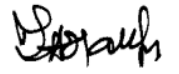

(b)

\section{Howh}

(c)

Fig 2: (a) Signature image, (b) Binary image, (c) Inverse of binary image

In image representation, pixels are the term used most widely to denote elements of a digital image [5]. In Figure 2(c) pixels containing 1's is the signature boundary which is our area of interest. The outer rectangular boundary which contains all 0 's in rows and column should be eliminated by the automatic cropping system as shown in Figure 3(a). We remove the noise and sharpen the edges of the cropped signature image by using median filter; it is an effective method that can suppress isolated noise without blurring sharp edges. There are also nonlinear neighborhood operations that can be performed for the purpose of noise reduction to do a better job of preserving edges than simple smoothing filters. In median filtering, the neighboring pixels are ranked according to brightness (intensity) and the median value becomes the new value for the central pixel [5]. Neighborhood averaging can suppress isolated out-of-range noise, but the side effect is that it also blurs sudden changes such as sharp edges. Specifically, the median filter replaces a pixel by the median of all pixels in the neighborhood.

$$
Y[m, n]=\operatorname{median}\{x[i, j],(i, j) \in w\}
$$

Where ' $w$ ' represents a neighborhood centered around location $(\mathrm{m}, \mathrm{n})$ in the image.

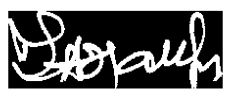

(a)

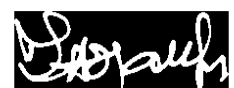

(b)
Fig 3: (a) Cropped signature image, (b) Filtered signature image

\section{FEATURE EXTRACTION}

Features of signature's image are classified into Global and Local features. Global features are extracted from a whole signature, based on all sample points in the input signature. Global features of the signature image can be extracted by the Hough transform, Discrete Cosine Transform (DCT), Discrete Radon Transform (DRT) etc. But in this paper we are using two methods i.e DRT and proposed for extracting the projection based features (Horizontal and Vertical projection).

The Radon transform is the projection of the image intensity along a radial line oriented at a specific angle. The Radon Transform of a function $f(x, y)$ denotes $g(s, \theta)$ is defined as its line integral along a line inclined at an angle $\theta$ from the $y$ axis and at a distance $\mathrm{s}$ from

$$
g(s, \theta)=\int_{-\infty}^{\infty} f(s \cos \theta-u \sin \theta, s \sin \theta+u \cos \theta) d u
$$

Where,

$$
\begin{aligned}
& \mathrm{s}=(\mathrm{x} \cos \theta+\mathrm{y} \sin \theta) \\
& \mathrm{u}=(-\mathrm{x} \sin \theta+\mathrm{y} \cos \theta)
\end{aligned}
$$

The radon function computes projections of an image matrix along specified directions [6][7]. The vertical projection feature vector which is one dimensional can be extracted by giving the 0 degree value to $\theta$ and 90 degree for horizontal projection feature vector. The vertical projection by DRT is shown in Figure (4).

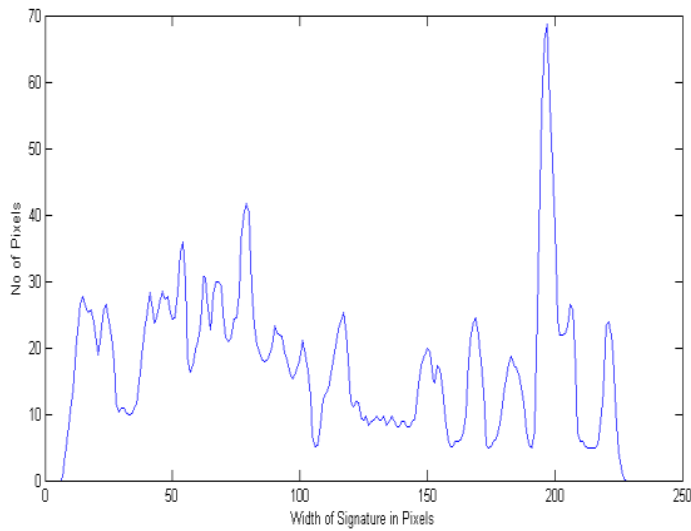

Fig 4: Vertical projections by DRT

In the proposed method, the filtered two dimensional signature's image contains 1's for signature and 0's for background as described in the previous section. First we find the matrix size of filtered image which is height (h) and width (w). For a particular X coordinate, whenever the values of all pixels along $\mathrm{Y}$ are summed up, it gives an estimate of the intensity of the signature at that $\mathrm{X}$ coordinate. The vertical projection of a signature is shown in Figure (5) which is also one dimensional. Following equation (3) describes about vertical projection (VP) [8].Similarly we can describes the horizontal projection which is defined by the equation (4).

$$
\begin{array}{ll}
\mathrm{VP}(\mathrm{X})=\sum_{\mathrm{Y}=1}^{\text {Heig ht }} \mathrm{P}(\mathrm{X}, \mathrm{Y}) & \mathrm{X} \leq \mathrm{w} \\
\mathrm{HP}(\mathrm{Y})=\sum_{\mathrm{X}=1}^{\text {Widt h }} \mathrm{P}(\mathrm{X}, \mathrm{Y}) & \mathrm{Y} \leq \mathrm{h}
\end{array}
$$




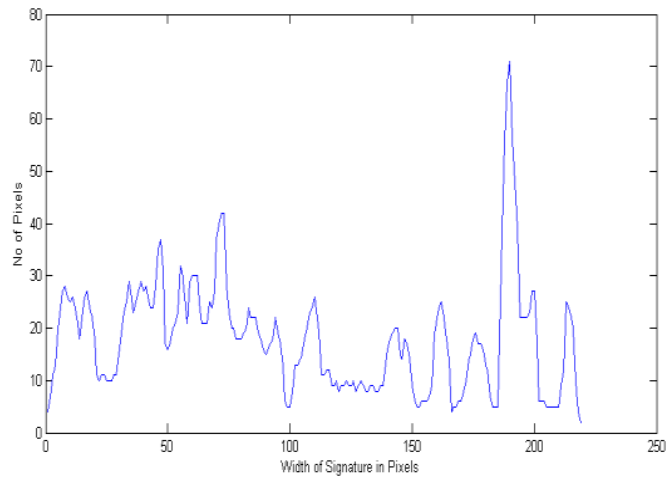

Fig 5: Vertical projection by proposed

\section{TRAINING}

Dynamic time warping algorithm using Euclidean distances between the features of the two points is used for alignment of the two signature's one dimensional feature vectors [9]. The Dynamic Time Warping gives the Total dissimilarity between the feature vectors which is proposed as the difference cost. This was first proposed by Sakoe and Chiba [10]. In order to understand DTW, two concepts need to be dealt with, features and distances. Distances have been classified as Local and Global. Local distance is a computational difference between a feature of one feature vector and a feature of another vector. Global distance is the overall computational difference between an entire feature vector and another feature vector of possibly different length. Suppose there are two feature vectors of signatures which are reference ' $x$ ' and test ' $y$ ' where $\mathrm{M}$ and $\mathrm{N}$ are the length ( or no of values ) of these feature vectors respectively and matching between these two vectors are performed by DTW algorithm. The reference and test vectors for DRT features and proposed features are shown in Figure 6 and 7 respectively. The Euclidean distance function is defined by $d(x, y)$ is given by equation (5).

$$
\mathrm{d}(\mathrm{x}, \mathrm{y})=\|\mathrm{x}-\mathrm{y}\| 2
$$

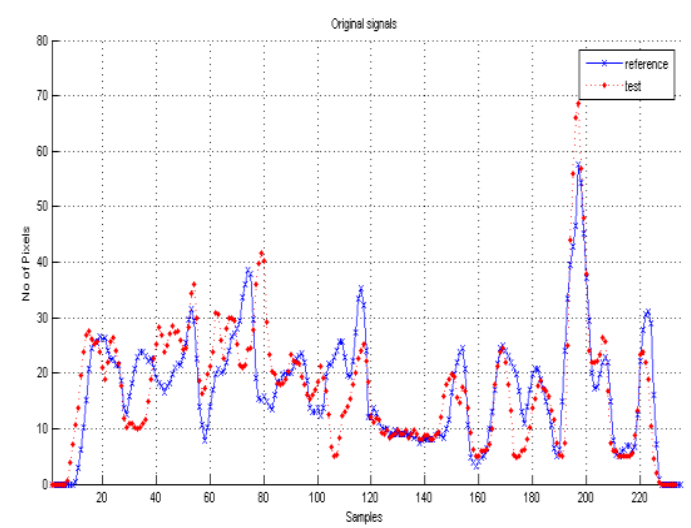

Fig 6: The reference and test vectors for DRT

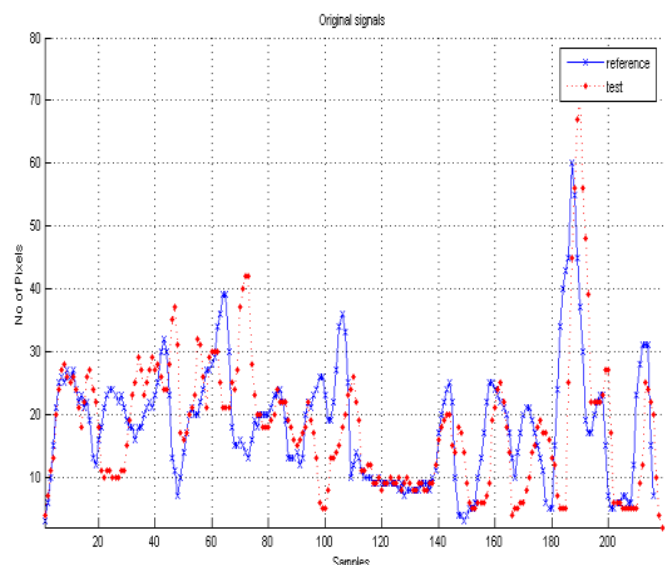

Fig 7: The reference and test vectors for proposed

Let $d(m, n)$ represents the cost associated with node $(m, n)$. The cost (local distance) of the starting node is represent by $\mathrm{d}$ $(1,1)$ which is assigned to zero and last node is represented by $(\mathrm{M}, \mathrm{N})$. The initial condition define as $\mathrm{D}(1,1)=\mathrm{d}(1,1)$ and $\mathrm{D}$ $(\mathrm{m}, \mathrm{n})$ is the global distance up to $(\mathrm{m}, \mathrm{n})$ and local distance at $(\mathrm{m}, \mathrm{n})$ is given by $\mathrm{d}(\mathrm{m}, \mathrm{n})$ and it is given by equation $(6)$

$$
\mathrm{D}(\mathrm{m}, \mathrm{n})=\mathrm{d}(\mathrm{m}, \mathrm{n})+\mathrm{D}(\mathrm{m}-1, \mathrm{n}-1)
$$

We can find least global distance by iterative process, given by equation (7) and DTW algorithm restricted by three conditions boundary, continuity and monotonic. The total dissimilarity between starting and last node is represented by $\mathrm{D}(\mathrm{M}, \mathrm{N})$ and matching between reference and test vectors by DRT and proposed are shown in Figure 8 and 9 respectively.

$$
\mathrm{D}(\mathrm{m}, \mathrm{n})=\mathrm{d}(\mathrm{m}, \mathrm{n})+\min [\mathrm{D}(\mathrm{m}-1, \mathrm{n}), \mathrm{D}(\mathrm{m}-1, \mathrm{n}-1), \mathrm{D}(\mathrm{m}, \mathrm{n}-
$$$$
\text { 1)] }
$$

$$
\text { DTWdist }=\mathrm{D}(\mathrm{M}, \mathrm{N})
$$

The training of the system for a particular person is performed in $\mathrm{K}$ number of genuine signature samples for that particular person [9][11][12]. The training of system is performed by equation (8) where $\mathrm{S} 1$ is the training score [8].

$$
\mathrm{S} 1=\frac{2}{\mathrm{~K}(\mathrm{~K}-1)} \sum_{\mathrm{i}=1}^{\mathrm{K}-1} \sum_{\mathrm{j}=\mathrm{i}+1}^{\mathrm{K}} \operatorname{DTW} \operatorname{dist}(\mathrm{Si}, \mathrm{Sj}), \quad \mathrm{i}, \mathrm{j} \leq \mathrm{K}
$$

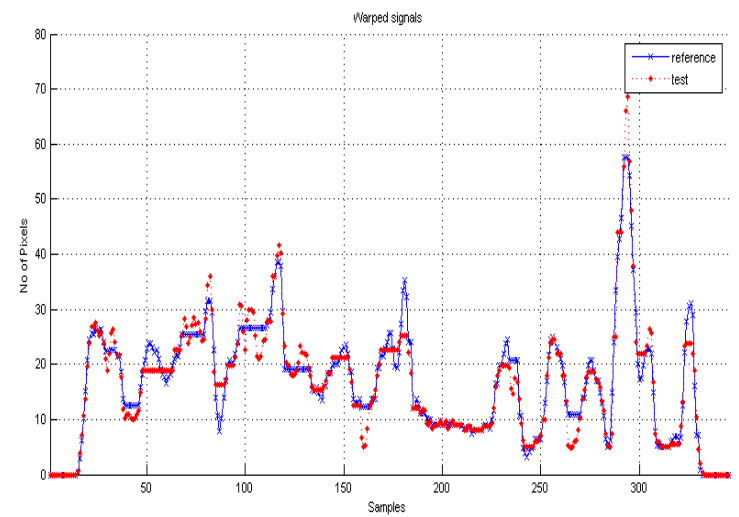

Fig 8: Matching warped signals between reference and test vectors by DRT 


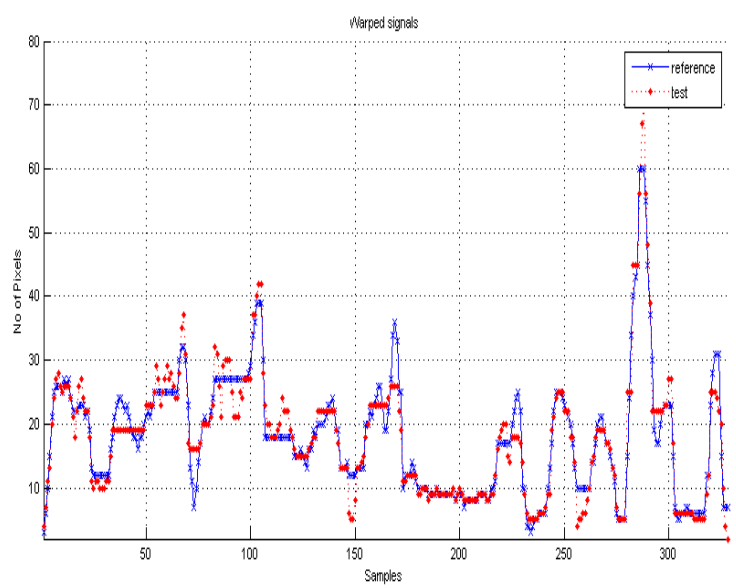

Fig 9: Matching warped signals between reference and test vectors by proposed

\section{VERIFICATION}

Verification of the signature is performed on test signature image St then it is decided whether signature is geunine or forgery. The test image is compared with each and every geunine signature samples which is used for training. The purpose is to compute the difference cost between two onedimensional feature vectors. Here we calculate the $\mathrm{K}$ difference cost between test signature image to each of the $\mathrm{K}$ genuine signature images $\mathrm{Si}$ and also calculate the mean of $\mathrm{K}$ difference cost [9][11]. In our signature verification system we used $\mathrm{K}=6$ number of genuine signatures. The verification of the system is performed by equation (9) where S2 is verification score [8].

$$
\mathrm{S} 2=\frac{1}{\mathrm{~K}} \sum_{\mathrm{i}=1}^{\mathrm{K}} \operatorname{DTW} \operatorname{dist}(\mathrm{St}, \mathrm{Si}), \quad \mathrm{i} \leq \mathrm{K}
$$

Now we calculate the score in equation (10). The decision to accept or reject is based on the value of the score.

$$
\text { Score }=\frac{\mathrm{S} 1}{\mathrm{~S} 2}
$$

\section{EXPERIMENTAL RESULT}

The experimentation is performed on our database which includes 550 genuine and 250 forgery signatures. For training purpose we use 126 genuine signatures with 6 genuine signatures of each person. All the individual signatures are scanned with 300 dpi resolution by a Canon Scan Lide 100 scanner and stored in JPEG format. The quality performance of the system is measured by false rejection rate (FRR), false acceptance rate (FAR) and equal error rate (EER). The FRR is the ratio of the number of genuine test signatures rejected to the total number of genuine test signatures used for testing. The FAR is the ratio of the number of forgeries accepted to the total number of forgeries used for testing. When the decision threshold is altered so as to decrease the FRR, the FAR will invariably increase, and vice versa. The Equal Error Rate (EER) of the system is defined as the probability of false acceptance and that of false rejection become equal for particular score. The EER of a system can be used to give a performance measure. The lower the EER is, the better is the system performance [13][14]. From the results shown in the Table-1 we can see that for the Vertical projection feature vectors using Discrete Radon transform, the threshold value of 0.71 gives FAR of 10.00 , FRR of 12.50 and minimum TER of 22.50 , that means it accept 25 forgery signature out of 250 and reject 53 genuine signature out of 424 , and we get equal error rate (EER) 11.79 for the score value 0.704 which is shown in Figure 10.

From the results shown in the Table- 2 we can see that for the Vertical projection feature vectors using Discrete Radon transform, the threshold value of 0.7 gives FAR of 8.80 , FRR of 8.25 and minimum TER of 17.05 , that means it accept 22 forgery signature out of 250 and reject 35 genuine signature out of 424, and we get equal error rate (EER) 8.40 for Score Value 0.7026 which is shown in Figure 11. In the system with DRT process time required for training our system for each person is between 0.6 to 1.5 seconds and by proposed method lies between 0.4 to 1.1 seconds. The proposed method also requires less memory for storage as compared to DRT method.

\section{CONCLUSION}

In this paper a comparative analysis is performed between the proposed method and DRT feature extraction method. A simple method of global feature extraction was proposed for off-line signature recognition system. From the Experimentation and testing results we found that Vertical projection based features by proposed method gives FRR, FAR, EER and TER which is less than Vertical projection based features by DRT because all feature vector values in proposed method are in integer form. The time required for training of the system and memory required for storage is less by proposed feature extraction method as compared to DRT method.

Table 1. Vertical Projection based Feature using DRT

\begin{tabular}{|c|c|c|c|}
\hline Score(Threshold) & FRR & FAR & TER \\
\hline 0.3 & 0.00 & 73.60 & 73.60 \\
\hline 0.4 & 0.23 & 48.80 & 49.03 \\
\hline 0.5 & 0.94 & 30.80 & 31.74 \\
\hline 0.6 & 3.53 & 19.20 & 22.73 \\
\hline 0.7 & 11.55 & 12.00 & 23.55 \\
\hline 0.71 & 12.50 & 10.00 & 22.50 \\
\hline 0.8 & 23.58 & 3.60 & 27.18 \\
\hline 0.9 & 38.67 & 0.80 & 39.47 \\
\hline 1.0 & 54.48 & 0.40 & 54.88 \\
\hline 1.1 & 73.82 & 0.00 & 73.82 \\
\hline 1.2 & 83.96 & 0.00 & 83.96 \\
\hline 1.3 & 92.92 & 0.00 & 92.92 \\
\hline
\end{tabular}

Table 2. Vertical Projection based Feature using Proposed Method

\begin{tabular}{|c|c|c|c|}
\hline Score(Threshold) & FRR & FAR & TER \\
\hline 0.3 & 0.00 & 80.40 & 80.40 \\
\hline 0.4 & 0.47 & 56.00 & 56.47 \\
\hline 0.5 & 0.94 & 35.60 & 36.54 \\
\hline 0.6 & 2.12 & 18.00 & 20.12 \\
\hline 0.7 & 8.25 & 8.80 & 17.05 \\
\hline 0.72 & 9.90 & 7.20 & 17.10 \\
\hline
\end{tabular}




\begin{tabular}{|l|l|l|l|}
\hline 0.8 & 20.75 & 2.40 & 23.15 \\
\hline 0.9 & 36.79 & 0.40 & 37.19 \\
\hline 1.0 & 56.13 & 0.40 & 56.53 \\
\hline 1.1 & 74.05 & 0.00 & 74.05 \\
\hline 1.2 & 85.84 & 0.00 & 85.84 \\
\hline 1.3 & 94.10 & 0.00 & 94.10 \\
\hline
\end{tabular}

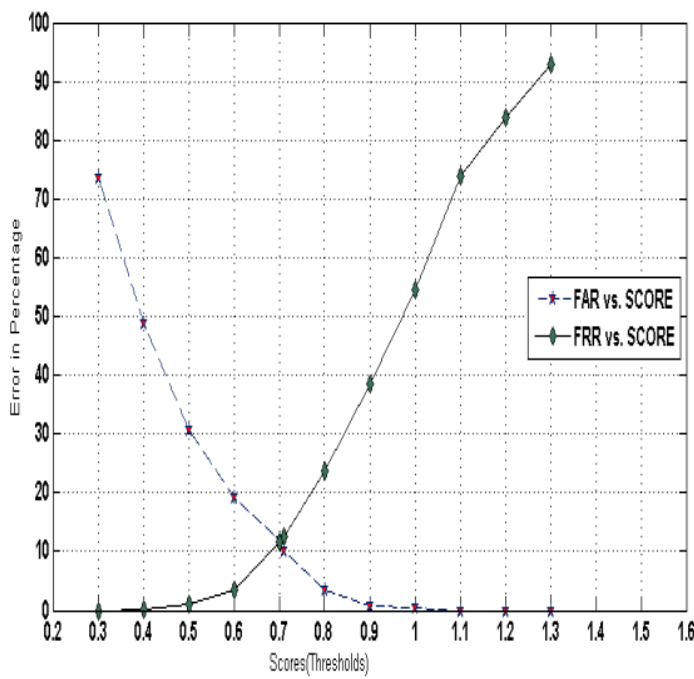

Fig 10: Equal Error Rate for Vertical projection based features using DRT when 6 genuine signatures are used for training

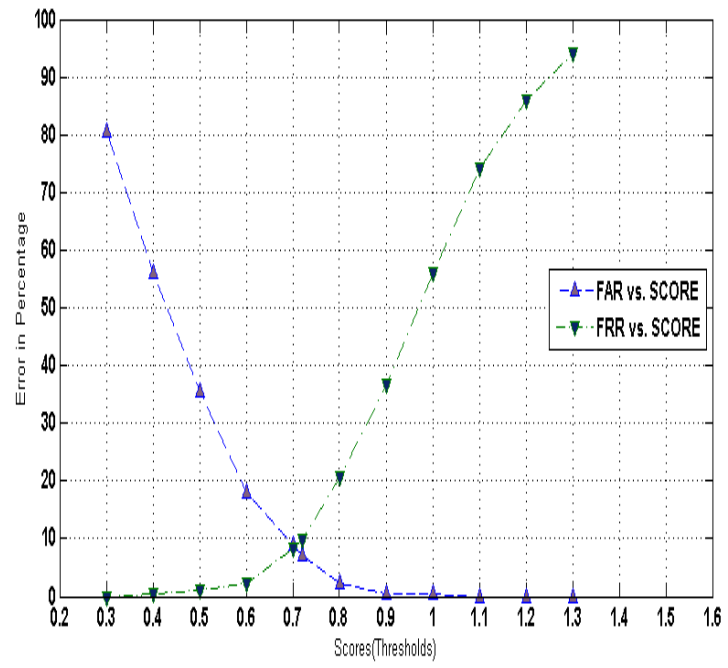

Fig 11: Equal Error Rate for Vertical projection based features using proposed method when 6 genuine signatures are used for training

\section{REFERENCES}

[1] Meenakshi S Arya, Vandana S Inamdar, "A Preliminary Study on Various Off-line Hand Written Signature Verification Approaches", International Journal of Computer Applications, vol. 1, no. 9, pp. 55-60, 2010.
[2] Richard O. Duda, Peter Hart, "Pattern Classification", 2nd edition, Wiley, 2010.

[3] Dipti Verma, Ankit Arora, "Preprocessing and Feature Extraction Method for Static Signature Recognition Using HoughTransform and Other Important Parameters", International Journal of Computer, Electronics \& Electrical Engineering,vol, 1 no.1, pp. 2126, December 2011

[4] Anil K. Jain, "Fundamentals of Digital Image Processing",2nd edition, Prentice Hall, 1988.

[5] Rafael C. Gonzalez, Richard E. Woods, "Digital Image Processing", 3rd edition, Prentice Hall, 2008.

[6] J. Coetzer, B. M. Herbst, J. A. du Preez, "Offline signature verification using the Discrete Radon Transform and a HiddenMarkovModel", EURASIP Journal on Applied Signal Processing, pp. 559-571, April 2004

[7] Vahid Kiani, Reza Pourreza and Hamid Reza Pourreza, "Offline Signature Verification Using Local Radon Transform and Support Vector Machines", International Journal of Image Processing (IJIP), vol 3, no.5, pp. 184194, 2009.

[8] Hifzan Ahmed, Shailja Shukla, "Global Features based Static Signature Verification system Using DTW", International Journal of Systems , Algorithms \& Applications, vol 2, Issue 4, pp. 13-17, April 2012.

[9] $\mathrm{Yu}$ Qiao and Chunjing $\mathrm{Xu}$ "Learning Mahalanobis Distance for DTW based Online Signature verification", Proceeding of the IEEE International Conference on Information and Automation Shenzhen, China, pp. 333338 , June 2011

[10] H. Sakoe and S. Chiba, "Dynamic programming algorithm optimization for spoken word recognition", Acoustics, Speech and Signal Processing, IEEE Transactions on, vol. 26, no. 1, pp. 43-49, February 1978.

[11] Kholmatov and B. Yanikoglu, "Identity authentication using improved online signature verification method," Pattern Recognition Letters, vol. 26, no. 15, pp. 2400 2408, November 2005

[12] Piyush Shanker, A.N. Rajagopalan, "Off-line signature verification using DTW", Pattern recognition letters, vol. 28, no. 12, pp. 1407-1414, September 2007.

[13] M. Taylan Das, L. Canan Dülger and H. Ergin Dülger, "Off-line Signature Verification (SV) using the Chisquare statistics", International Journal Biometrics, vol. 3, no. 1, 2011.

[14] Muhammad Reza Pourshahabi, Mohamad Hoseyn Sigari, Hamid Reza Pourreza, "Offline handwritten signature Identification and verification using Contourlet Transform", International Conference of Soft Computing and Pattern Recognition, IEEE computer society, pp 670-673, December 2009. 